\title{
On the $V$-filtration of $\mathcal{D}$-modules
}

\author{
Nero Budur \\ Department of Mathematics, Johns Hopkins University, Baltimore, MD \\ 21218-2686, U.S.A. \\ nbudur@math. jhu .edu
}

Summary. In this mostly expository note we give a down-to-earth introduction to the $V$-filtration of M. Kashiwara and B. Malgrange on $\mathcal{D}$-modules. We survey some applications to generalized Bernstein-Sato polynomials, multiplier ideals, and monodromy of vanishing cycles.

The $V$-filtration on $\mathcal{D}$-modules was introduced by $\mathrm{M}$. Kashiwara and B. Malgrange to construct vanishing cycles in the category of (regular holonomic) $\mathcal{D}$-modules. Our aim is to give a down-to-earth introduction to this notion and describe some applications. The first application is to the generalized Bernstein-Sato polynomials introduced in [3]. Following G. Lyubeznik, we extend a finiteness result on the set of these polynomials. Then we describe applications to multiplier ideals [4], [3] and to monodromy of vanishing cycles and Hodge spectrum [2], [4].

Acknowledgments. We thank M. Mustaţă who provided us with preliminary notes on the $V$-filtration. We thank M. Saito for clarifications on many issues. Most of what I learned about the $V$-filtration is from discussions with them and with L. Ein.

\section{Basics}

In this section we introduce the filtration $V$ and prove a few consequences assuming its existence. For a complete account of the $V$-filtration consult [12], [5], [8], [15].

Let $X$ be a smooth complex variety. The sheaf $\mathcal{D}_{X}$ of algebraic differential operators on $X$ is generated locally by multiplication by functions and by the tangent vector fields. If $X=\mathbb{A}^{n}$ is the affine $n$-space, then $\mathcal{D}_{X}$ is the Weyl algebra

$$
A_{n}(\mathbb{C})=\mathbb{C}\left[x_{1}, \ldots, x_{n}, \partial_{1}, \ldots, \partial_{n}\right]
$$


where $\partial_{i}=\partial / \partial x_{i}$ and $\partial_{i} x_{j}-x_{j} \partial_{i}=\delta_{i, j}$. In these notes we will consider only left $\mathcal{D}_{X}$-modules, the most important for our applications being $\mathcal{O}_{X}$. We will frequently work locally without specifically assuming that $X$ is affine.

The $V$-filtration of M. Kashiwara and B. Malgrange on $\mathcal{D}_{X}$-modules is defined with respect to some closed subvariety $Z \subset X$.

Case $Z \subset X$ smooth. First we consider smooth closed subvarieties $Z \subset X$. Let $\mathcal{I} \subset \mathcal{O}_{X}$ denote the ideal of $Z$. In local coordinates, write

$$
X=\{(x, t)\}, Z=\{x\}=\{t=0\},
$$

with $x=x_{1}, \ldots, x_{n}$, and $t=t_{1}, \ldots, t_{r}$. Then

$$
\mathcal{D}_{X}=\mathbb{C}\left[x, t, \partial_{x}, \partial_{t}\right] .
$$

The $V$-filtration on $\mathcal{D}_{X}$ is defined by

$$
V^{j} \mathcal{D}_{X}=\left\{P \in \mathcal{D}_{X} \mid P \mathcal{I}^{i} \subset \mathcal{I}^{i+j} \text { for all } i \in \mathbb{Z}\right\},
$$

with $j \in \mathbb{Z}$ and $\mathcal{I}^{i}=\mathcal{O}_{X}$ for $i \leq 0$. Locally,

$$
V^{j} \mathcal{D}_{X}=\sum_{|\beta|-|\gamma| \geq j} h_{\alpha, \beta, \gamma}(x) \partial_{x}^{\alpha} t^{\beta} \partial_{t}^{\gamma} .
$$

Here we use vectorial indices for monomials, and $|\beta|=\sum_{i} \beta_{i}$. A computation with local coordinates shows:

(i) $V^{j_{1}} \mathcal{D}_{X} \cdot V^{j_{2}} \mathcal{D}_{X} \subset V^{j_{1}+j_{2}} \mathcal{D}_{X}$, with equality if $j_{1}, j_{2} \geq 0$;

(ii) $V^{j} \mathcal{D}_{X}=\mathcal{I}^{j} \cdot V^{0} \mathcal{D}_{X} \cdot \mathcal{D}_{X,-j}=\mathcal{D}_{X,-j} \cdot V^{0} \mathcal{D}_{X} \cdot \mathcal{I}^{j}$, where $\mathcal{D}_{X, j} \subset \mathcal{D}_{X}$ are the operators of order $\leq j$, and $\mathcal{I}^{j}=\mathcal{D}_{X, j}=\mathcal{O}_{X}$ for $j \leq 0$.

Definition 1.1. The filtration $V$ along $Z$ on a coherent left $\mathcal{D}_{X}$-module $M$ is an exhaustive decreasing filtration of coherent $V^{0} \mathcal{D}_{X}$-submodules $V^{\alpha}:=$ $V^{\alpha} M$, such that:

(i) $\left\{V^{\alpha}\right\}_{\alpha}$ is indexed left-continuously and discretely by rational numbers, i.e., $V^{\alpha}=\cap_{\beta<\alpha} V^{\beta}$, every interval contains only finitely many $\alpha$ with $G r_{V}^{\alpha} \neq 0$, and these $\alpha$ must be rational. Here, $G r_{V}^{\alpha}=V^{\alpha} / V^{>\alpha}$, where $V^{>\alpha}=\cup_{\beta>\alpha} V^{\beta}$.

(ii) $t_{j} V^{\alpha} \subset V^{\alpha+1}$, and $\partial_{t_{j}} V^{\alpha} \subset V^{\alpha-1}$ for all $\alpha \in \mathbb{Q}$, i.e., $\left(V^{i} \mathcal{D}_{X}\right)\left(V^{\alpha} M\right) \subset$ $V^{\alpha+i} M$

(iii) $\sum_{j} t_{j} V^{\alpha}=V^{\alpha+1}$ for $\alpha \gg 0$;

(iv) the action of $\sum_{j} \partial_{t_{j}} t_{j}-\alpha$ on $G r_{V}^{\alpha}$ is nilpotent on $X$.

All conditions are independent of the choice of local coordinates.

Theorem 1.2 (M. Kashiwara, B. Malgrange). The filtration $V$ along $Z$ exists if $M$ is regular holonomic and quasi-unipotent. 
It is beyond our scope to introduce the theory of holonomic systems of differential operators with regular singularities (see [1], [6]). It suffices to say that all the $\mathcal{D}$-modules considered in the applications are regular holonomic and quasi-unipotent.

\section{Proposition 1.3. The $V$-filtration along $Z$ is unique.}

Proof. Let $\widetilde{V}$ be another filtration on $M$ satisfying Definition 1.1. By symmetry, it suffices to show that $V^{\alpha} \subset \widetilde{V}^{\alpha}$ for every $\alpha$. Suppose that $\alpha \neq \beta$ and consider

$$
V^{\alpha} \cap \tilde{V}^{\beta} /\left(V^{>\alpha} \cap \tilde{V}^{\beta}\right)+\left(V^{\alpha} \cap \tilde{V}^{>\beta}\right) .
$$

Since both filtrations satisfy Definition 1.1-(iv), both $\left(\sum_{j} \partial_{t_{j}} t_{j}-\alpha\right)$ and $\left(\sum_{j} \partial_{t_{j}} t_{j}-\beta\right)$ are nilpotent on this module. Hence the module is zero.

We show now that for every $\alpha$ we have

$$
V^{\alpha} \subset V^{>\alpha}+\widetilde{V}^{\alpha}
$$

Fix $w \in V^{\alpha}$. By exhaustion, there is $\beta \ll 0$ (in particular $\beta<\alpha$ ) such that $w \in \widetilde{V}^{\beta}$. By what we have already proved, we may write $w=w_{1}+w_{2}$, with $w_{1} \in V^{>\alpha}$ and $w_{2} \in V^{\alpha} \cap \widetilde{V}^{>\beta}$. If we replace $w$ by $w_{2}$, then the class in $V^{\alpha} / V^{>\alpha}$ remains unchanged, but we may choose a larger $\beta$. We can repeat the process as long as $\beta<\alpha$. Since the $\widetilde{V}$-filtration is discrete, we can repeat the process until we have $\beta \geq \alpha$. Hence the class of $w$ in $V^{\alpha} / V^{>\alpha}$ can be represented by an element in $\widetilde{V}^{\alpha}$, and we get (1).

Since the $V$-filtration is discrete, a repeated application of (1) shows that for every $\beta \geq \alpha$ we have $V^{\alpha} \subset V^{\beta}+\widetilde{V}^{\alpha}$. We deduce from Definition 1.1-(iii) that if we fix $\beta \gg 0$, then

$$
V^{\alpha} \subset \mathcal{I}^{q} \cdot V^{\beta}+\widetilde{V}^{\alpha}
$$

for big enough $q$, where $\mathcal{I} \subset \mathcal{O}_{X}$ is the ideal pf $Z$. By coherence, $V^{\beta}=$ $\sum V^{0} \mathcal{D}_{X} \cdot u_{i}$ for finitely many $u_{i}$. By exhaustion, there exists some $\gamma \in \mathbb{Z}$ such that $\widetilde{V}^{\gamma}$ contains the $u_{i}$, hence also $V^{\beta}$. By Definition 1.1-(ii), for $q$ with $q+\gamma \geq \alpha$ we have $\mathcal{I}^{q} \widetilde{V}^{\gamma} \subset \widetilde{V}^{\alpha}$. Thus $\mathcal{I}^{q} V^{\beta} \subset \widetilde{V}^{\alpha}$. Hence by (2) we have $V^{\alpha} \subset \widetilde{\widetilde{V}}^{\alpha}$.

Case $Z \subset X$ arbitrary. Now let $X$ be a smooth complex variety and $Z \neq X$ a closed subscheme. Suppose $f_{1}, \ldots, f_{r} \in \mathcal{O}_{X}$ generate the ideal $\mathcal{I} \subset \mathcal{O}_{X}$ of $Z$. Let $i: X \rightarrow X \times \mathbb{A}^{r}=Y$ be the embedding $x \mapsto\left(x, f_{1}(x), \ldots, f_{r}(x)\right)$. Let $t_{j}: Y \rightarrow \mathbb{A}^{1}$ be the projection with $t_{j} \circ i=f_{j}$.

Let $N$ be a $\mathcal{D}_{X}$-module and $M=i_{*} N$, where $i_{*}$ is the direct image for left $D$-modules. Working out the definition of the direct image (e.g., [1]), one gets $M=N \otimes \mathbb{C}\left[\partial_{t_{1}}, \ldots, \partial_{t_{r}}\right]$ with the left $\mathcal{D}_{Y}$-action given as follows. Let $x_{1}, \ldots, x_{n}$ be local coordinates on $X$. For $g \in \mathcal{O}_{X}, m \in N$, and $\partial_{t}^{\nu}=\partial_{t_{1}}^{\nu_{1}} \ldots \partial_{t_{r}}^{\nu_{r}}$, 


$$
\begin{aligned}
& g\left(m \otimes \partial_{t}^{\nu}\right)=g m \otimes \partial_{t}^{\nu}, \quad \partial_{x_{i}}\left(m \otimes \partial_{t}^{\nu}\right)=\partial_{x_{i}} m \otimes \partial_{t}^{\nu}-\sum_{j} \frac{\partial f_{j}}{\partial x_{i}} m \otimes \partial_{t_{j}} \partial_{t}^{\nu}, \\
& \partial_{t_{j}}\left(m \otimes \partial_{t}^{\nu}\right)=m \otimes \partial_{t_{j}} \partial_{t}^{\nu}, \quad t_{j}\left(m \otimes \partial_{t}^{\nu}\right)=f_{j} m \otimes \partial_{t}^{\nu}-\nu_{j} m \otimes\left(\partial_{t}^{\nu}\right)_{j},
\end{aligned}
$$

where $\left(\partial_{t}^{\nu}\right)_{j}$ is obtained from $\partial_{t}^{\nu}$ by replacing $\nu_{j}$ with $\nu_{j}-1$. If $N$ satisfies the requirements of Theorem 1.2, then also $M$ does.

Definition 1.4. The $V$-filtration along $Z$ on $N(=N \otimes 1)$ is defined by $V^{\alpha} N=$ $(N \otimes 1) \cap V^{\alpha} M$, for $\alpha \in \mathbb{Q}$ and $V$ on $M$ taken along $X \times\{0\}$.

Proposition 1.5. The definition above depends on the ideal $\mathcal{I}$ of $Z$ in $X$ and not on the particular generators chosen.

Proof. (cf. [3]-2.7) Suppose $g_{1}, \ldots, g_{r^{\prime}} \in \mathcal{O}_{X}$ also generate $\mathcal{I}$, with $g_{j}=$ $\sum a_{i j} f_{i}, a_{i j} \in \mathcal{O}_{X}$. Let $i^{\prime}: Y=X \times \mathbb{A}^{r} \rightarrow Y^{\prime}=X \times \mathbb{A}^{r} \times \mathbb{A}^{r^{\prime}}$ be the embedding sending $(x, t)$ to $\left(x, t, t^{\prime}\right)$, where $t_{j}^{\prime}=\sum a_{i j}(x) t_{i}, j=1, \ldots, r^{\prime}$. The crucial fact here is that the image of $X \times\{0\}$ is $X \times\{0\} \times\{0\}$. Working locally, we can assume that $x, t, u$ is a local coordinate system on $Y^{\prime}$ such that $Y=\{u=0\}, X=\{t=u=0\}$. Hence $M^{\prime}=i_{*}^{\prime} M$ can be written as $M \otimes \mathbb{C}\left[\partial_{u_{1}}, \ldots, \partial_{u_{r^{\prime}}}\right]$ with left $\mathcal{D}_{Y^{\prime}}$-action as above. Note that some simplifications occur: $\partial_{x_{i}}\left(m \otimes \partial_{u}^{\nu}\right)=\partial_{x_{i}} m \otimes \partial_{u}^{\nu}, \partial_{t_{i}}\left(m \otimes \partial_{u}^{\nu}\right)=\partial_{t_{i}} m \otimes \partial_{u}^{\nu}$, and $u_{j}\left(m \otimes \partial_{u}^{\nu}\right)=-\nu_{j} m \otimes\left(\partial_{u}^{\nu}\right)_{j}$, where $m \in M, \partial_{u}^{\nu}=\partial_{u_{1}}^{\nu_{1}} \ldots \partial_{u_{r^{\prime}}}^{\nu^{\prime}}$, and $\left(\partial_{u}^{\nu}\right)_{j}$ is obtained from $\partial_{u}^{\nu}$ by replacing $\nu_{j}$ with $\nu_{j}-1$.

The claim follows if we show that

$$
V^{\alpha} M^{\prime}=\sum_{\nu \in \mathbb{N}^{r^{\prime}}} V^{\alpha+|\nu|} M \otimes \mathbb{C}\left[\partial_{u}^{\nu}\right]
$$

is the $V$-filtration on $M^{\prime}$ along $X \times\{0\} \times\{0\}$.

Let us check the axioms for the $V$-filtration. In local coordinates, $V^{0} \mathcal{D}_{Y^{\prime}}$ is generated over $\mathcal{O}_{Y^{\prime}}$ by the $\partial_{x_{i}}$, and the $v \partial_{w}$ with $v, w \in\left\{t_{1}, \ldots, t_{r}, u_{1}, \ldots, u_{r^{\prime}}\right\}$. From definition, these actions are well-defined on $V^{\alpha} M^{\prime}$. To show that $V^{\alpha} M^{\prime}$ is coherent over $V^{0} \mathcal{D}_{Y^{\prime}}$, it is enough to show that $V^{\alpha} M^{\prime}$ is locally finitely generated since $V^{0} \mathcal{D}_{Y^{\prime}}$ is coherent. Since $V^{\alpha} M$ is locally finitely generated over $V^{0} \mathcal{D}_{Y}$, we have that for $c \gg 0, \sum_{|\nu| \leq c} V^{\alpha+|\nu|} M \otimes \mathbb{C}\left[\partial_{u}^{\nu}\right]$ is locally finitely generated. Also for $c \gg 0, V^{\alpha+c+1} M=\sum_{i} t_{i} V^{\alpha} M$ by the axiom (iii) of Definition 1.1. Therefore the rest of $V^{\alpha} M^{\prime}$ is recovered from $\sum_{|\nu| \leq c}$ through the action of the $t_{i} \partial_{u_{j}}$, hence $V^{\alpha} M^{\prime}$ is finitely generated.

The axioms (ii) and (iii) of Definition 1.1 follow from the definition of $V^{\alpha} M^{\prime}$, the simplifications noted above in the $\mathcal{D}_{Y^{\prime}}$-action on $M^{\prime}$, and the same axioms applied to $V^{\alpha} M$.

The last property to show is the nilpotency of $s-\alpha$ on $\operatorname{Gr}_{V}^{\alpha} M^{\prime}$, where $s=\sum_{i} \partial_{t_{i}} t_{i}+\sum_{j} \partial_{u_{j}} u_{j}$. Let $m \otimes \partial_{u}^{\nu} \in V^{\alpha} M^{\prime}$ with $m \in V^{\alpha+|\nu|} M$. Then

$$
(s-\alpha)\left(m \otimes \partial_{u}^{\nu}\right)=\left(\sum_{i} \partial_{t_{i}} t_{i}-|\nu|-\alpha\right) m \otimes \partial_{u}^{\nu} .
$$


Hence $(s-\alpha)^{k}\left(m \otimes \partial_{u}^{\nu}\right) \in V^{\alpha+1} M^{\prime}$ if $k$ is the nilpotency order of $\left(\sum_{i} \partial_{t_{i}} t_{i}-\right.$ $(\alpha+|\nu|))$ on $\operatorname{Gr}_{V}^{\alpha+|\nu|} M$.

Examples will be provided in Section 3.

\section{Bernstein-Sato polynomials}

The $V$-filtration can be applied to show the existence of quite general Bernstein-Sato polynomials, [3]. See [6] for an account of the classical version of these polynomials. Following G. Lyubeznik [11], we prove a finiteness result on the set of all polynomials that are Bernstein-Sato polynomials in a sense we make precise later.

We keep the notation from the previous section. Suppose first that $Z \subset X$ is a smooth closed subvariety. To keep this article as concise as possible we take Theorem 1.2 for granted. Then the quickest way to proceed is by means of the following technical tool.

Definition 2.1. Let $M$ be a coherent left $\mathcal{D}_{X}$-module. For $u \in M$, the Bernstein-Sato polynomial $b_{u}(s)$ of $u$ is the monic minimal polynomial of the action of $s=-\sum_{j} \partial_{t_{j}} t_{j}$ on $V^{0} \mathcal{D}_{X} u / V^{1} \mathcal{D}_{X} u$.

We suppressed from the notation the fact that $b_{u}(s)$ also depends on $Z$. Then we can make explicit the $V$-filtration as follows.

Proposition 2.2 (C. Sabbah [15]). If the $V$-filtration along $Z$ exists on $M$, then $b_{u}(s)$ exists, it is non-zero for all $u \in M$, and has rational coefficients. Moreover

$$
V^{\alpha} M=\left\{u \in M \mid \alpha \leq c \text { if } b_{u}(-c)=0\right\} .
$$

Proof. Suppose first that $u \in V^{\alpha} M$. Recall that $\sum_{j} \partial_{t_{j}} t_{j}-\beta$ is nilpotent on $V^{\beta} / V^{>\beta}$ and $V$ is indexed discretely. Then, for a given $\beta$ there is a polynomial $b(s)$ depending on $\beta$, having all roots $\leq-\alpha$ (and rational), and such that $b\left(-\sum_{j} \partial_{t_{j}} t_{j}\right) \cdot u \in V^{\beta}$. Hence it is enough to show that there is $\beta$ such that $V^{\beta} \cap V^{0} \mathcal{D}_{X} u \subset V^{1} \mathcal{D}_{X} u$.

Let $A=\bigoplus_{i \geq 0} V^{i} \mathcal{D}_{X} \tau^{-i}$ and define $F_{k}(A)=\bigoplus_{i \geq 0}\left(V^{i} \mathcal{D}_{X} \cap \mathcal{D}_{X, k}\right) \tau^{-i}$. Then by Lemma 2.3, $A$ is a noetherian ring. Now $\bigoplus_{i \geq 0} V^{i} M$ is coherent over $A$ because by axiom (iii) of Definition 1.1, there exists $i_{0}$ such that $V^{i} M$ is recovered from $V^{i_{0}} M$ if $i \geq i_{0}$. Denote by $N$ the $V^{0} \mathcal{D}_{X}$-submodule $V^{0} \mathcal{D}_{X} u$, and let $U^{i}=V^{i} \cap N$ for $i \geq 0$. Then $\bigoplus_{i \geq 0} U^{i} N$ is also coherent over $A$ since $A$ is noetherian. It follows that $\bigoplus_{i>0} \operatorname{Gr}_{U}^{i} N$ is coherent over $\bigoplus_{i>0} \operatorname{Gr}_{V}^{i} \mathcal{D}_{X}$, in particular locally finitely generated. If $i$ is big compared with the degrees of local generators, we see that $U^{i} N \subset V^{1} \mathcal{D}_{X} u$.

Conversely, fix an element $u \in M$ and suppose that $\alpha \leq c$ whenever $b_{u}(-c)=0$. Let $\alpha_{u}=\max \left\{\beta \mid u \in V^{\beta}\right\}$. We need to show that $\alpha \leq \alpha_{u}$. It is 
enough to show that $b_{u}\left(-\alpha_{u}\right)=0$. For $\beta \neq \alpha_{u},\left(\sum_{j} \partial_{t_{j}} t_{j}-\beta\right)$ is invertible on $V^{\alpha_{u}} / V^{>\alpha_{u}}$. But $b_{u}\left(-\sum_{j} \partial_{t_{j}} t_{j}\right) u \in V^{>\alpha_{u}}$. Hence we must have $b_{u}\left(-\alpha_{u}\right)=0$.

Lemma 2.3 ([6]-A.29). Let $A$ be a be a filtered ring (sheaf on $X)$. Assume that $F_{0}(A)$ and $\operatorname{Gr}^{F}(A)$ are noetherian rings, and that $G r_{k}^{F}(A)$ are (locally) finitely generated $F_{0}(A)$-modules for all $k$. Then $A$ is noetherian.

Now let $Z \subset X$ be an arbitrary closed subset. Let $f_{1}, \ldots, f_{r}$ be generators of the ideal of $Z$, where $f_{j} \neq 0$ for any $j$. Then $\mathcal{D}_{X}$ acts naturally on $\mathcal{O}_{X}\left[\prod_{i} f_{i}^{-1}, s_{1}, \ldots, s_{r}\right] \prod_{i} f_{i}^{s_{i}}$, where the $s_{i}$ are independent variables. Define a $\mathcal{D}_{X}$-linear action of $t_{i}$ by $t_{i}\left(s_{j}\right)=s_{j}+1$ if $i=j$, and $t_{i}\left(s_{j}\right)=s_{j}$ otherwise. Let $s_{i j}=s_{i} t_{i}^{-1} t_{j}$, and $s=\sum_{i} s_{i}$. We will see in Lemma 2.6 that under a well-defined isomorphism the $t_{i}$ 's here correspond to the $t_{i}$ 's introduced in the second part of Section 1 .

Definition $2.4([3])$. The Bernstein-Sato polynomial $b_{f}(s)$ of $f:=\left(f_{1}, \ldots, f_{r}\right)$ is defined to be the monic polynomial of the lowest degree in s satisfying the relation

$$
b_{f}(s) \prod_{i} f_{i}^{s_{i}}=\sum_{j}\left(P_{j} f_{j} \prod_{i} f_{i}^{s_{i}}\right),
$$

where the $P_{j}$ belong to the ring generated by $\mathcal{D}_{X}$ and the $s_{i j}$. For $h \in \mathcal{O}_{X}$, define similarly $b_{f, h}(s)$ with $\prod_{i} f_{i}^{s_{i}}$ replaced by $\prod_{i} f_{i}^{s_{i}} h$.

Example 2.5.

(i) $f=x^{2}+y^{3}$. Then $b_{f}(s)=(s+1)(s+5 / 6)(s+7 / 6)$ and

$$
P=\left(\partial_{y}^{3} / 27+y \partial_{x}^{2} \partial_{y} / 6+x \partial_{x}^{3} / 8\right) .
$$

(ii) $f=\left(x_{2} x_{3}, x_{1} x_{3}, x_{1} x_{2}\right)$. Then $b_{f}(s)=(s+3 / 2)(s+2)^{2}$ and the $s_{i j}$ cannot be avoided by the operators $P_{j}$ in the above definition (see [3]).

The polynomial $b_{Z}(s):=b_{f}(s-r)$ with $r=\operatorname{codim}_{X} Z$ is shown in [3] to depend only on $Z$ and not on $f$. The existence of non-zero $b_{f, h}(s)$ follows from the following.

Lemma 2.6. With the notation as in Definition 1.4, if $M=i_{*} \mathcal{O}_{X}, u=h \otimes 1$ with $h \in \mathcal{O}_{X}$, and the $V$-filtration is taken along $X \times\{0\}$, then $b_{u}(s)=b_{f, h}(s)$.

Proof. It suffices to show that $b_{u}(s)$ is the minimal polynomial of the action of $s=\sum_{j} s_{j}$ on

$$
\mathcal{D}_{X}\left[s_{i j}\right] \prod_{j} f_{j}^{s_{j}} h / \sum_{k} \mathcal{D}_{X}\left[s_{i j}\right] f_{k} \prod_{j} f_{j}^{s_{j}} h,
$$

a quotient of submodules of $\mathcal{O}_{X}\left[\prod_{i} f_{i}^{-1}, s_{1}, \ldots, s_{r}\right] \prod_{i} f_{i}^{s_{i}} h$. We can check that $\mathcal{D}_{X}\left[s_{i j}\right] \prod_{j} f_{j}^{s_{j}} h$ and $\sum_{k} \mathcal{D}_{X}\left[s_{i j}\right] f_{k} \prod_{j} f_{j}^{s_{j}} h$ are isomorphic to $V^{0} \mathcal{D}_{Y} u$ and $V^{1} \mathcal{D}_{Y} u$. The action of $t_{j}$ is defined by $s_{j} \mapsto s_{j}+1, \prod_{j} f_{j}^{s_{j}} h$ corresponds to $u, s_{j}$ corresponds to $-\partial_{t_{j}} t_{j}$, and $s_{i j}=s_{i} t_{i}^{-1} t_{j}$. 
Hence it follows from Proposition2.2 that $b_{f, h}(s)$ are polynomials with rational coefficients. One is allowed to change the field of definition in the coefficients of the $f_{j}$ 's.

Proposition 2.7. There exist non-zero Bernstein-Sato polynomials $b_{f, h}(s)$ even if in Definition 2.4 one replaces $X, \mathcal{O}_{X}$, and $\mathcal{D}_{X}$ with $\mathbb{A}_{k}^{n}, k\left[x_{1}, \ldots, x_{n}\right]$, and $A_{n}(k)$ (the Weyl algebra) respectively, for $k$ a field of characteristic zero.

Proof. First, suppose that the coefficients of the $f_{j}$ 's lie in a subfield $K$ of $\mathbb{C}$. Then also the scalar coefficients of the $P_{j}$ 's can be assumed to lie in $K$. Indeed, (3) implies, after equating coefficients of monomials in $s_{i}$ 's and $x_{i}$ 's, that certain $K$-linear relations $(*)$ hold among the scalar coefficients of the $P_{j}$ 's. Let $L$ be the field generated by the coefficients of the $P_{j}$ 's. Fix a basis $S$ of $L / K$ containing 1 and such that every scalar coefficient $c$ which appears in a $P_{j}$ can be written as a unique $K$-linear combination of a finite number of elements of $S$. Let $c_{1} \in K$ be the coefficient of 1 in $c$ under this basis, and let $P_{j, 1}$ be the induced operator. Then the $K$-linear relations $(*)$ hold with $c_{1}$ replacing $c$, and so $(3)$ holds with $P_{j, 1}$ replacing $P_{j}$.

Now, going back to our proposition, the conclusion follows from the Lefschetz principle. Indeed, let $K$ be a subfield of $k$ generated over $\mathbb{Q}$ by the coefficients of the $f_{j}$ 's. Since $\mathbb{C}$ has infinite transcendental dimension over $\mathbb{Q}$, $K$ can be embedded into $\mathbb{C}$. Then the coefficients of the $P_{j}$ are in $K \subset k$.

We extend a result of G. Lyubeznik [11] to the case of these more general Bernstein-Sato polynomials. The proof follows closely his proof.

Proposition 2.8. Fix $n$ and $d$ positive integers. The set of all polynomials which are of the form $b_{f}(s)$ for some $f=f_{1}, \ldots, f_{r} \in k\left[x_{1}, \ldots, x_{n}\right]$ with $\operatorname{deg} f_{i} \leq d$ is finite even if $k$ is varying over all the fields of characteristic zero.

Proof. Let $N$ be the number of monomials in $x_{1}, \ldots, x_{n}$ of degree $\leq d$. Then the $f$ 's are the closed $k$-rational points of $\left[\mathbb{A}_{k}^{N}\right]^{\times r}$. Let $P=\sum_{|\alpha| \leq d} c_{\alpha} x^{\alpha}$ be the polynomial of $n$ variables of degree $d$ with undetermined coefficients. Then $B_{k}=k\left[\text { the } c_{\alpha}{ }^{\prime} s\right]^{\otimes r}$ is the coordinate ring of $\left[\mathbb{A}_{k}^{N}\right]^{\times r}$. Define

$$
F_{i}=1 \otimes \cdots \otimes P \otimes \cdots \otimes 1 \in B_{\mathbb{Q}}\left[x_{1}, \ldots, x_{n}\right]
$$

by placing $P$ in the $i$-th position. Here $\times$ and $\otimes$ mean over $\mathbb{Q}$.

Let $Y$ be a reduced and irreducible closed subset of $\left[\mathbb{A}_{\mathbb{Q}}^{N}\right]^{\times r}$. Denote by $G=\left(G_{i}\right)_{i}$ the image of $F=\left(F_{i}\right)_{i}$ under the natural $\mathbb{Q}$-algebra homomorphism $B_{\mathbb{Q}}\left[x_{1}, \ldots, x_{n}\right] \rightarrow \mathbb{Q}(Y)\left[x_{1}, \ldots, x_{n}\right]$, where $\mathbb{Q}(Y)$ is the function field of $Y$. Let $\mathbb{Q}[Y]$ be the coordinate ring of $Y$. Then, we have a functional equation

$$
b_{G}(s) \prod_{i} G_{i}^{s_{i}}=\sum_{j} P_{j} G_{j} \prod_{i} G_{i}^{s_{i}}
$$


with $s=\sum s_{i}$ and $P_{j} \in A_{n}(\mathbb{Q}(Y))\left[\right.$ the $s_{i j}$ 's]. Denote by $c \in \mathbb{Q}[Y]$ the common denominator. Denote by $U(c) \subset Y$ the subscheme whose coordinate ring is $\mathbb{Q}[Y]_{c}$. By specializing (4), the $f$ 's given by the closed $k$-rational points of $U(c) \times k$ have $b$-functions $b_{f}(s)$ dividing $b_{G}(s)$. Hence they are only finitely many such $b_{f}(s)$ even if $k$ varies.

We proceed now by induction on the dimension of $Y$ proving that the $k$-rational points of $Y \times k$ give only finitely many $b$-functions even if $k$ varies. For dimension zero, $c=1$ and so $U(c)=Y$. In higher dimensions, $Y \backslash U(c)$ is the union of reduced and irreducible closed subsets of smaller dimension.

\section{Multiplier ideals}

The multiplier ideals introduced by A. Nadel [14] encode the complexity of singularities via their resolutions. It turns out that they are essentially the same as the $V$ filtration on $\mathcal{O}_{X}$.

Let $X$ be a smooth complex variety and $Z \neq X$ a closed subscheme. Let $\mu: X^{\prime} \rightarrow X$ be a $\log$ resolution of $(X, Z)$. That is $\mu$ is proper birational, $X^{\prime}$ is smooth, and $\operatorname{Ex}(\mu) \cup \mu^{-1} Z$ is a divisor with simple normal crossings. Here $\operatorname{Ex}(\mu)$ denotes the exceptional locus of $\mu$. Let $\mathcal{I} \subset \mathcal{O}_{X}$ denote the ideal of $Z$. Let $H$ be the effective divisor on $X^{\prime}$ such that $\mu^{-1}(\mathcal{I}) \cdot \mathcal{O}_{X^{\prime}}=\mathcal{O}_{X^{\prime}}(-H)$.

Definition 3.1. For $\alpha>0$, the multiplier ideal of $(X, \alpha \cdot Z)$ is defined as

$$
\mathcal{J}(\alpha \cdot Z)=\mu_{*}\left(\omega_{X^{\prime} / X} \otimes \mathcal{O}_{X^{\prime}}(-\llcorner\alpha \cdot H\lrcorner)\right) .
$$

Here $\omega_{X^{\prime} / X}=\operatorname{det} \Omega_{X^{\prime}}^{1} \otimes \mu^{*}\left(\operatorname{det} \Omega_{X}^{1}\right)^{\vee}$ is the sheaf of relative top-dimensional forms, and $\llcorner$.$\lrcorner rounds down the coefficients of the irreducible divisors. One can$ extend this definition to a formal combination of closed subschemes $\sum_{i} \alpha_{i} \cdot Z_{i}$ by replacing $\alpha \cdot H$ with $\sum_{i} \alpha_{i} \cdot H_{i}$.

The original analytic definition of multiplier ideals is, locally,

$$
\mathcal{J}(\alpha \cdot Z)=\left\{\left.h \in \mathcal{O}_{X}|| h\right|^{2} /\left(\sum_{1 \leq i \leq r}\left|f_{i}\right|^{2}\right)^{\alpha} \in L_{l o c}^{1}\right\},
$$

where $f_{1}, \ldots, f_{r}$ generate $\mathcal{I}$. The first definition shows the second is independent of the choice of generators, and the second definition shows the first is independent of the choice of resolution. See [9] for more on multiplier ideals.

The multiplier ideals measure how singular $Z$ is. The intuition here is that smaller multiplier ideals means worse singularities. For example, varying the coefficient in front of $Z$, one obtains a decreasing family $\{\mathcal{J}(\alpha \cdot Z)\}_{\alpha \in \mathbb{Q}}$. Because of the rounding-down of coefficients in the construction of $\mathcal{J}(\alpha \cdot Z)$ there exist positive rational numbers $0<\alpha_{1}<\alpha_{2}<\cdots$ such that

$$
\mathcal{J}\left(\alpha_{j} \cdot Z\right)=\mathcal{J}(\alpha \cdot Z) \neq \mathcal{J}\left(\alpha_{j+1} \cdot Z\right)
$$


for $\alpha_{j} \leq \alpha<\alpha_{j+1}$ where $\alpha_{0}=0$. These numbers $\alpha_{j}(j>0)$ are called the jumping numbers of the multiplier ideals associated to $(X, Z)$. The $\log$-canonical threshold of $(X, Z)$ is the smallest non-zero jumping number, $\operatorname{lc}(X, Z)=\alpha_{1}$. Equivalently, $\operatorname{lc}(X, Z)$ is the number $\alpha$ such that $\mathcal{J}(\alpha \cdot Z) \neq$ $\mathcal{O}_{X}$, but $\mathcal{J}((\alpha-\epsilon) Z)=\mathcal{O}_{X}$ for $0<\epsilon \ll 1$.

\section{Example 3.2.}

(i) $Z=\left\{x^{2}+y^{3}=0\right\} \subset \mathbb{A}^{2}$. Then $\mathcal{J}(\alpha \cdot Z)$ is equal to $\mathcal{O}_{X}$ if $0<\alpha<5 / 6$, and it is the maximal ideal at $(0,0)$ if $5 / 6 \leq \alpha<1$.

(ii) $Z=\left\{x_{1} x_{2}=x_{2} x_{3}=x_{1} x_{3}=0\right\} \subset \mathbb{A}^{3}$. Then $\mathcal{J}(\alpha \cdot Z)$ is equal to $\mathcal{O}_{X}$ if $0<\alpha<3 / 2$, and it is the ideal $\left(x_{1}, x_{2}, x_{3}\right)$ if $3 / 2 \leq \alpha<2$. This follows from [9]-III.9.3.4 which gives the formula for multiplier ideals of monomial ideals.

(iii) The multiplier ideals of hyperplane arrangements, and more generally, stratified locally conical divisors are determined in [13], and respectively [16].

Theorem $3.3([3],[4])$. For $\alpha>0, V^{\alpha} \mathcal{O}_{X}=\mathcal{J}((\alpha-\epsilon) \cdot Z)$, where $0<\epsilon \ll 1$ and the filtration $V$ of $\mathcal{O}_{X}$ is taken along $Z$ as in Definition 1.4.

The relation with Bernstein-Sato polynomials is then given by Proposition 2.2 and Lemma 2.6:

Corollary 3.4. For $\alpha>0$,

$$
\mathcal{J}(\alpha \cdot Z)=\left\{h \in \mathcal{O}_{X} \mid \alpha<c \text { if } b_{f, h}(-c)=0\right\},
$$

where $f=f_{1}, \ldots, f_{r}$ is any set of generators of the ideal $\mathcal{I} \subset \mathcal{O}_{X}$ of $Z$.

In particular,

$$
\operatorname{lc}(X, Z)=-\left(\text { biggest root of } b_{f}(s)\right),
$$

since $b_{f}(s)=b_{f, 1}(s)$ by definition [7], [10].

\section{Monodromy of vanishing cycles}

The initial scope of the $V$-filtration of M. Kashiwara and B. Malgrange was to construct vanishing cycles in the category of (regular holonomic) $\mathcal{D}$-modules.

Let $X$ be a smooth complex variety. Denote by $M_{r h}\left(\mathcal{D}_{X}\right)$ the abelian category of regular holonomic $\mathcal{D}_{X}$-modules, and by $D_{r h}^{b}\left(\mathcal{D}_{X}\right)$ the derived category of bounded complexes of $\mathcal{D}_{X}$-modules with regular holonomic cohomology. By A. Beilinson, this is equivalent with the bounded derived category of $M_{r h}\left(\mathcal{D}_{X}\right)$. Let $D_{c}^{b}(X)$ be the derived category of bounded complexes of sheaves (in the analytic topology of $X$ ) of $\mathbb{C}$-vector spaces with constructible cohomology. The Riemann-Hilbert correspondence generalizing the analogy between the $\mathcal{D}_{X}$-module $\mathcal{O}_{X}$ and the constant sheaf $\mathbb{C}_{X}$ states (see [1]): 
Theorem 4.1 (M. Kashiwara, Z. Mebkhout). Let $X$ be a smooth complex variety. There is a well-defined functor

$$
D R: D_{r h}^{b}\left(\mathcal{D}_{X}\right) \longrightarrow D_{c}^{b}(X)
$$

which is an equivalence of categories commuting with the usual six functors. $D R$ also defines an equivalence $M_{r h}\left(\mathcal{D}_{X}\right) \rightarrow \operatorname{Perv}(X)$, where Perv $(X) \subset$ $D_{c}^{b}(X)$ is the subcategory of perverse sheaves.

Let $f \in \mathcal{O}_{X}$ be a regular function. The vanishing cycles functor $\phi_{f}$ on $D_{c}^{b}(X)$ and the monodromy action $T$ on it should then have a meaning only in terms of $\mathcal{D}$-modules since the shift $\phi_{f}[-1]$ restricts as a functor to Perv $(X)$. Let $M$ be a regular holonomic $\mathcal{D}_{X}$-module. Let $\tilde{M}$ be its direct image under the graph of $f$, as in section 2. If $M$ is also quasi-unipotent, then there exists a $V$-filtration indexed by $\mathbb{Q}$ on $\tilde{M}$ along $X \times\{0\}$. If $M$ is not quasi-unipotent, a close version of the following still holds:

Theorem 4.2 (M. Kashiwara, B. Malgrange). Let $\alpha \in[0,1)$ be a rational number. $G r_{V}^{\alpha} \tilde{M}$ corresponds to the $\exp (-2 \pi i \alpha)$-eigenspace of $\phi_{f}[-1](D R(M))$ with respect to the action of the semisimple part $T_{s}$ of the monodromy.

Combining this result with Theorem 3.3 and with additional structures such as mixed Hodge modules, one obtains a relation between multiplier ideals and the Hodge spectrum of hypersurface singularities. Let $f: X \rightarrow \mathbb{A}^{1}$ be a regular function. Recall that if $i_{x}: x \hookrightarrow f^{-1}(0)$ is a point, the Milnor fiber of $f$ at $x$ is is the Milnor fiber of the corresponding holomorphic germ $f:\left(\mathbb{C}^{m}, 0\right) \rightarrow(\mathbb{C}, 0)$,

$$
M_{f, x}=\left\{z \in \mathbb{C}^{m}|| z \mid<\epsilon \text { and } f(z)=t\right\}
$$

for a fixed $t$ with $0<|t|<\epsilon \ll 1$. Then

$$
H^{i}\left(i_{x}^{*} \phi_{f} \mathbb{C}_{X}\right)=\tilde{H}^{i}\left(M_{f, x}, \mathbb{C}\right),
$$

where $\tilde{H}$ stands for reduced cohomology. These vector spaces are endowed with the monodromy action $T$ and with mixed Hodge structures on which $T_{s}$ acts as automorphism. Indeed, the mixed Hodge module theory of M. Saito on the left-hand side of (5) recovers the mixed Hodge structure of V. Navarro-Aznar from the right-hand side. As numerical invariants encoding the behaviour of the Hodge filtration $F$ under $T_{s}$ one has the generalized equivariant Euler characteristics

$$
n(i, \alpha)=\sum_{j}(-1)^{j} \operatorname{dim} \operatorname{Gr}_{F}^{i} \tilde{H}^{j}\left(M_{f, x}, \mathbb{C}\right)_{\alpha},
$$

where $\alpha \in \mathbb{Q} \cap[0,1), i \in\{0, \ldots, m-1\}$, and the subscript $\alpha$ stands for the eigenspace of $T_{s}$ with eigenvalue $\exp (2 \pi i \alpha)$. These invariants form the Hodge spectrum of $f$ introduced by J. Steenbrink [17]. For $\alpha \in(0,1]$, let 


$$
n_{\alpha, x}(f)=(-1)^{n-1} n(m-1,1-\alpha),
$$

so that $n_{\alpha, x}(f)$ describe the spectrum for the smallest piece of the Hodge filtration.

Example 4.3. If $f=x^{2}+y^{3}$ and $x=(0,0) \in \mathbb{A}^{2}$, then $n_{\alpha, x}(f)$ is zero for $\alpha \notin\{5 / 6,7 / 6\}$, and is 1 otherwise.

On the other hand, for every jumping number $\alpha \in(0,1]$ of $(X, Z)$ where $Z$ is the zero set of a regular function $f$, define the inner jumping multiplicity at $x$

$$
\left.n_{\alpha, x}(Z)=\operatorname{dim} \mathcal{J}((1-\epsilon) \alpha \cdot Z) / \mathcal{J}((1-\epsilon) \alpha \cdot Z)+\delta \cdot x\right),
$$

where $0<\epsilon \ll \delta \ll 1$. It is proved in [2] that $n_{\alpha, x}(Z)$ is finite and does not depend on $\epsilon$ and $\delta$. Let $\widetilde{\mathcal{O}_{X}}$ be the $\mathcal{D}$-module direct image of $\mathcal{O}_{X}$ under the graph of $f$. In connection with Theorem 3.3 it is crucial to observe that the smallest piece of the Hodge filtration on $V^{\alpha} \widetilde{\mathcal{O}_{X}}$ is exactly $V^{\alpha} \mathcal{O}_{X}$. Then the above arguments lead to:

Theorem 4.4 ([2], [4]). For $\alpha \in(0,1]$,

$$
n_{\alpha, x}(f)=n_{\alpha, x}(Z) .
$$

\section{References}

1. A. Borel, P.-P. Grivel, B. Kaup, A. Haefliger, B. Malgrange and F. Ehlers - Algebraic D-modules, Perspectives in Mathematics, vol. 2, Academic Press Inc., Boston, MA, 1987.

2. N. Budur - "On Hodge spectrum and multiplier ideals", Math. Ann. 327 (2003), no. 2, p. 257-270.

3. N. Budur, M. Mustaţă and M. SAito - "Bernstein-Sato polynomials of arbitrary varieties", 2004, preprint.

4. N. Budur and M. SAITO - "Multiplier ideals, V-filtration, and spectrum", to appear in J. Algebraic Geom.

5. M. KASHIWARA - "Vanishing cycle sheaves and holonomic systems of differential equations", Algebraic geometry (Tokyo/Kyoto, 1982), Lecture Notes in Math., vol. 1016, Springer, Berlin, 1983, p. 134-142.

6. M. Kashiwara - D-modules and microlocal calculus, Translations of Mathematical Monographs, vol. 217, American Mathematical Society, Providence, RI, 2003.

7. J. Kollár - "Singularities of pairs", Algebraic geometry - Santa Cruz 1995, Proc. Sympos. Pure Math., vol. 62, Amer. Math. Soc., Providence, RI, 1997, p. $221-287$.

8. G. LAUMON - "Transformations canoniques et spécialisation pour les $D$-modules filtrés", Astérisque (1985), no. 130, p. 56-129, Differential systems and singularities (Luminy, 1983).

9. R. LAZARSFELD - "Positivity in algebraic geometry", book to appear in 2004 . 
10. B. Lichtin - "Poles of $|f(z, w)|^{2 s}$ and roots of the $b$-function", Ark. Mat. 27 (1989), no. 2, p. 283-304.

11. G. Lyubeznik - "On Bernstein-Sato polynomials", Proc. Amer. Math. Soc. 125 (1997), no. 7, p. 1941-1944.

12. B. Malgrange - "Polynômes de Bernstein-Sato et cohomologie évanescente", Analysis and topology on singular spaces, II, III (Luminy, 1981), Astérisque, vol. 101, Soc. Math. France, Paris, 1983, p. 243-267.

13. M. Mustaţă - "Multiplier ideals of hyperplane arrangements", 2004, math. AG/0402232.

14. A. M. NADEL - "Multiplier ideal sheaves and Kähler-Einstein metrics of positive scalar curvature", Ann. of Math. (2) 132 (1990), no. 3, p. 549-596.

15. C. Saвbah - "D-modules et cycles évanescents (d'après B. Malgrange et M. Kashiwara)", Géométrie algébrique et applications, III (La Rábida, 1984), Travaux en Cours, vol. 24, Hermann, Paris, 1987, p. 53-98.

16. M. SAITO - "Multiplier ideals, $b$-function, and spectrum", 2004.

17. J. H. M. SteEnBRINK - "The spectrum of hypersurface singularities", Astérisque (1989), no. 179-180, p. 11, 163-184, Actes du Colloque de Théorie de Hodge (Luminy, 1987). 